\title{
On Multi-Index Mittag-Leffler Function of Several Variables and Fractional Differential Equations
}

\author{
B. B. Jaimini $\mathbb{D}^{1},{ }^{1}$ Manju Sharma $\left(\mathbb{D},{ }^{1}\right.$ D. L. Suthar $\mathbb{D},{ }^{2}$ and S. D. Purohit $\mathbb{D D}^{3}$ \\ ${ }^{1}$ Department of Mathematics, Government College, Kota 324001, Rajasthan, India \\ ${ }^{2}$ Department of Mathematics, Wollo University, P. O. Box: 1145, Amhara, Ethiopia \\ ${ }^{3}$ Department of HEAS (Mathematics), Rajasthan Technical University, Kota 324001, Rajasthan, India
}

Correspondence should be addressed to D. L. Suthar; dlsuthar@gmail.com

Received 2 May 2021; Accepted 30 September 2021; Published 18 October 2021

Academic Editor: Zakia Hammouch

Copyright (C) 2021 B. B. Jaimini et al. This is an open access article distributed under the Creative Commons Attribution License, which permits unrestricted use, distribution, and reproduction in any medium, provided the original work is properly cited.

\begin{abstract}
In this paper, we have studied a unified multi-index Mittag-Leffler function of several variables. An integral operator involving this Mittag-Leffler function is defined, and then, certain properties of the operator are established. The fractional differential equations involving the multi-index Mittag-Leffler function of several variables are also solved. Our results are very general, and these unify many known results. Some of the results are concluded at the end of the paper as special cases of our primary results.
\end{abstract}

\section{Introduction}

Recently, Mittag-Leffler (M-L) functions have demonstrated their special connection to fractional calculus, with a particular emphasis on fractional calculus problems arising from implementations. Several new special functions and implementations have been discovered over the last few decades. The advancement of research in the new era of special functions and their applications in mathematical modelling continues to attract many scientists from various disciplines (see recent papers; [1-13]).

The Mittag-Leffler function is extended to multi-index function in the following form $[14,15]$ :

$$
E_{\gamma, K}\left[\left(\alpha_{1}, \beta_{1}\right), \ldots,\left(\alpha_{m}, \beta_{m}\right) ; z\right]=\sum_{n=0}^{\infty} \frac{(\gamma)_{K n} z^{n}}{\prod_{j=1}^{m} \Gamma\left(\beta_{j}+n \alpha_{j}\right) n !}
$$

where $\alpha_{j}, \beta_{j}, \gamma \in \mathbb{C} ; \mathfrak{R}\left(\alpha_{j}\right)>0 ; \mathfrak{R}\left(\beta_{j}\right)>0 \quad(j=1, \ldots, m)$; $\mathfrak{R}\left(\sum_{j=1}^{m} \alpha_{j}\right)>0$; and $K$ is an arbitrary complex number, i.e., $K \in \mathbb{C}$.

If we make $\gamma=K=1 \mathrm{in}(1)$ it reduces to the multi-index M-L function studied by Kiryakova [16, 17].

A multivariable extension of Mittag-Leffler function widely studied by Gautam [18], and also by Saxena et al. ([19], p. 547, Equation (7.1)), is defined and represented as follows: 


$$
E_{\left(\rho_{r}\right), \lambda}^{\left(\gamma_{r}\right),\left(l_{r}\right)}\left(z_{1}, \ldots, z_{r}\right)=\sum_{k_{1}, \ldots k_{r}=0}^{\infty} \frac{\left(\gamma_{1}\right)_{l_{1} k_{1}} \ldots\left(\gamma_{r}\right)_{l_{r} k_{r}}}{\Gamma\left(\lambda+k_{1} \rho_{1}+\cdots+k_{r} \rho_{r}\right)} \frac{z_{1}^{k_{1}} \ldots z_{r}^{k_{r}}}{k_{1} ! \ldots k_{r} !}
$$

where $\lambda, \gamma_{j}, l_{j}, \rho_{j} \in \mathbb{C} \mathfrak{R}\left(\rho_{j}\right)>0 ; \mathfrak{R}\left(l_{j}\right)>0 ; \lambda \notin \mathbb{Z}_{0}^{-}=\{0,-1$, $-2, \ldots\}$; and $j=1,2, \ldots, r$.

Motivated by the work on these functions, we consider here the subsequent multivariable and multi-index Mittag-Leffler function:

$$
E_{\left(\rho_{1}^{(r)}\right), \ldots,\left(\rho_{m}^{(r)}\right) ; \beta_{1}, \ldots, \beta_{m}}^{\left(\gamma_{r}\right),\left(l_{r}\right)}\left[z_{1}, \ldots, z_{r}\right]=\sum_{k_{1}, \ldots, k_{r}=0}^{\infty} \frac{\prod_{i=1}^{r}\left(\gamma_{i}\right)_{l_{i} k_{i}}}{\prod_{j=1}^{m} \Gamma\left(\beta_{j}+\sum_{i=1}^{r} \rho_{j}^{(i)} k_{i}\right)} \frac{z_{1}^{k_{1}}}{k_{1} !} \ldots \frac{z_{r}^{k_{r}}}{k_{r} !},
$$

where $\beta_{j}, \gamma_{i}, l_{i}, \rho_{j}^{(i)} \in \mathbb{C} ; \mathfrak{R}\left(\rho_{j}^{(i)}\right)>0 ; \mathfrak{R}\left(l_{i}\right)>0 ; \quad \beta_{j} \notin \mathbb{Z}_{0}^{-}=$ $\{0,-1,-2, \ldots\} ;\left(\rho_{j}^{(r)}\right) \equiv \rho_{j}^{\prime}, \rho_{j}^{\prime \prime}, \ldots, \rho_{j}^{(r)} ; i=1, \ldots, r ;$ and $j=$ $1, \ldots, m$.
We have also studied here, the integral operator involving the function defined by (3), as follows:

$$
\begin{aligned}
& \left(E_{\left(\rho_{1}^{(r)}\right), \ldots,\left(\rho_{m}^{(r)}\right) ; \beta_{1}, \ldots, \beta_{m} ; \omega_{r} ; a+}^{\left(\gamma_{r}\right),\left(l_{r}\right)} \psi\right)(x) \\
= & \int_{a}^{x}(x-t)^{\beta_{1}-1} E_{\left(\rho_{1}^{(r)}\right), \ldots,\left(\rho_{m}^{(r)}\right) ; \beta_{1}, \ldots, \beta_{m}}^{\left(\gamma_{r}\right),\left(l_{r}\right)}\left[\omega_{1}(x-t)^{\rho_{1}^{\prime}}, \ldots, \omega_{r}(x-t)^{\rho_{1}^{(r)}}\right] \psi(t) \mathrm{d} t,
\end{aligned}
$$

with $\omega_{i}, \rho_{j}^{(i)}, \gamma_{i}, l_{i}, \beta_{j} \in \mathbb{C} ; x>a ;\left|\omega_{i} \quad(x-t)^{\rho_{1}^{(i)}}\right|<1$; $\mathfrak{R}\left(\rho_{j}^{(i)}\right)>0 ; j=1, \ldots, m$; and $i=1, \ldots, r$.

The Riemann-Liouville fractional derivative operator $D_{0+}^{\alpha}$ is defined as follows [20]:

$$
\left(D_{a+}^{\alpha} \psi\right)(x)=\left(\frac{d}{\mathrm{~d} x}\right)^{n}\left(I_{a+}^{n-\alpha} \psi\right)(x),(\alpha \in \mathbb{C} ; \mathfrak{R}(\alpha)>0 ; n=|\Re(\alpha)|+1),
$$

where $\left(I_{a+}^{\alpha} \psi\right)(x)$ is the fractional integral operator defined by

$$
\left(I_{a+}^{\alpha} \psi\right)(x)=\frac{1}{\Gamma(\alpha)} \int_{a}^{x} \frac{\psi(t)}{(x-t)^{1-\alpha}} \mathrm{d} t
$$

The elementary definitions are also required to be mentioned as follows.

The Laplace transform of fractional derivative $\left(D_{0+}^{\alpha} f\right)(x)$ is given as

$$
\mathfrak{L}\left(D_{0+}^{\alpha} f ; s\right)=s^{\alpha} F(s)-\sum_{k=1}^{n} s^{k-1} D_{0+}^{\alpha-k} f(0+),(\Re(s)>0 ; \quad(n-1<\alpha<n)) .
$$

Also, the formula for Laplace transform is

$$
\frac{d^{n}}{\mathrm{~d} s^{n}}[\mathbf{Q}\{y(x):(s)\}]=(-1)^{n} \mathfrak{Q}\left[x^{n} y(x)\right](s) .
$$

\section{Results Required}

The integral for the generalized M-L function defined in (3) is given by

$$
\begin{aligned}
& \frac{1}{\Gamma(\sigma)} \int_{0}^{x}(x-t)^{\beta_{1}-1} t^{\sigma-1} E_{\left(\rho_{1}^{(r)}\right), \ldots,\left(\rho_{m}^{(r)}\right) ; \beta_{1}, \ldots, \beta_{m}}^{\left(\gamma_{r}\right),\left(l_{r}\right)}\left[\omega_{1}(x-t)^{\rho_{1}^{\prime}}, \ldots, \omega_{r}(x-t)^{\rho_{1}^{(r)}}\right] \mathrm{d} t \\
& =x^{\beta_{1}+\sigma-1} E_{\left(\rho_{1}^{(r)}\right), \ldots,\left(\rho_{m}^{(r)}\right) ; \beta_{1}+\sigma, \beta_{2}, \ldots, \beta_{m}}^{\left(\gamma_{r}\right),\left(l_{r}\right)}\left[\omega_{1} x^{\rho_{1}^{\prime}}, \ldots, \omega_{r} x^{\rho_{1}^{(r)}}\right] .
\end{aligned}
$$


The result in (9) is established in view of definition in (3) and using the elementary beta integral.
The Laplace transform of $E_{\left(\rho_{1}^{(r)}\right), \ldots,\left(\rho_{m}^{(r)}\right) ; \beta_{1}, \ldots, \beta_{m}}^{\left(\gamma_{r}\right),\left(l_{r}\right)}$ $\left(\omega_{1} x^{\rho_{1}^{\prime}}, \ldots, \omega_{r} x^{\rho_{1}^{(r)}}\right)$ defined in (3), easily obtained here, is as follows:

$$
\begin{aligned}
& \mathcal{Q}\left\{x^{\beta_{1}-1} E_{\left(\rho_{1}^{(r)}\right), \ldots,\left(\rho_{m}^{(r)}\right) ; \beta_{1}, \ldots, \beta_{m}}^{\left(\gamma_{r}\right),\left(l_{r}\right)}\left[\omega_{1} x^{\rho_{1}^{\prime}}, \ldots, \omega_{r} x^{\rho_{1}^{(r)}}\right]\right\}(x)=s^{-\beta_{1}} \prod_{j=2}^{m}\left(\frac{1}{\Gamma\left(\beta_{j}\right)}\right)
\end{aligned}
$$

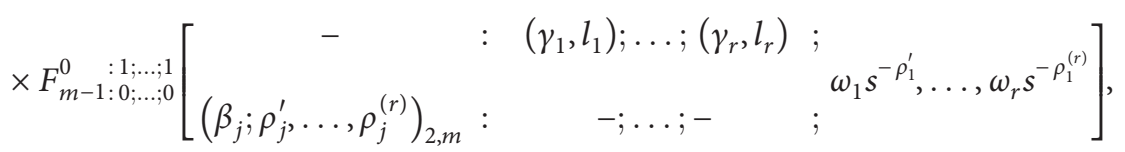

where $\alpha, \beta_{j}, \rho_{j}^{(i)}, \gamma_{i}, l_{i}, \omega_{i} \in \mathbb{C} ; \mathfrak{R}(s)>0 ; \mathfrak{R}\left(\rho_{j}^{(i)}\right)>0 ; \mathfrak{R}\left(\beta_{j}\right)$; $\Re\left(l_{i}\right)>0 ; j=1, \ldots, m ; i=1, \ldots, r ; \quad$ and $\quad\left(\beta_{j}: \rho_{j}^{\prime}, \ldots\right.$, $\left.\rho_{j}^{(r)}\right)_{2, m} \equiv\left(\beta_{2} ; \rho_{2}^{\prime}, \ldots, \rho_{2}(r)\right), \ldots,\left(\beta_{m} ; \rho_{m}^{\prime}, \ldots, \rho_{m}^{(r)}\right)$.

Here, $F\left[\omega_{1} s^{-\rho_{1}^{\prime}}, \ldots, \omega_{r} s^{-\rho_{1}^{(r)}}\right]$ is the generalized Lauricella function ([21], p. 37, Equations (21-23)).

\section{Main Results}

Theorem 1. Let $a \in \mathbb{R}_{+} ; \alpha, \beta_{j}, \rho_{j}^{(i)}, \gamma_{i}, l_{i}, \omega_{i} \in \mathbb{C} ; \mathfrak{R}$ $(\alpha)>0 ; \Re\left(\rho_{j}^{(i)}\right)>0 ; \Re$ $\left(\beta_{j}\right)>0 ;$ and $\Re\left(l_{i}\right)>0(j=1, \ldots, m ; i=1, \ldots, r)$. Then, for $x>a$, we have

$$
\begin{aligned}
& D_{a+}^{\alpha}\left[(t-a)^{\beta_{1}-1} E_{\left(\begin{array}{l}
\left.\rho_{1}^{(r)}\right), \ldots,\left(\rho_{m}^{(r)}\right) ; \beta_{1}, \ldots, \beta_{m} \\
\left(\gamma_{r}\right),\left(l_{r}\right)
\end{array}\right.}^{\alpha}\left\{\omega_{1}(t-a)^{\rho_{1}^{\prime}}, \ldots, \omega_{r}(t-a)^{\rho_{1}^{(r)}}\right\}\right](x) \\
& =(x-a)^{\beta_{1}-\alpha-1} E_{\left(\rho_{1}^{(r)}\right), \ldots,\left(\rho_{m}^{(r)}\right) ; \beta_{1}-\alpha, \beta_{2}, \ldots, \beta_{m}}^{\left(\gamma_{r}\right),\left(l_{r}\right)}\left[\omega_{1}(x-a)^{\rho_{1}^{\prime}}, \ldots, \omega_{r}(x-a)^{\rho_{1}^{(r)}}\right]
\end{aligned}
$$

and

$$
\begin{aligned}
& I_{a+}^{\alpha}\left[(t-a)^{\beta_{1}-1} E_{\begin{array}{c}
\left(\gamma_{r}\right),\left(l_{r}\right) \\
\left(\rho_{1}^{(r)}\right), \ldots,\left(\rho_{m}^{(r)}\right) ; \beta_{1}, \ldots \beta_{m}
\end{array}}\left\{\omega_{1}(t-a)^{\rho_{1}^{\prime}}, \ldots, \omega_{r}(t-a)^{\rho_{1}^{(r)}}\right\}\right](x) \\
= & (x-a)^{\beta_{1}+\alpha-1} E_{\left(\begin{array}{l}
\left(\gamma_{r}\right),\left(l_{r}\right) \\
\left(\rho_{1}^{(r)}\right), \ldots,\left(\rho_{m}^{(r)}\right) ; \beta_{1}+\alpha, \beta_{2}, \ldots, \beta_{m}
\end{array}\right.}\left[\omega_{1}(x-a)^{\rho_{1}^{\prime}}, \ldots, \omega_{r}(x-a)^{\rho_{1}^{(r)}}\right] . \\
\mathcal{R}(\alpha)>0 ; \Re\left(\rho_{j}^{(i)}\right)>0 ; \Re\left(\beta_{j}\right)>0 ; & \left(D_{0+}^{\alpha} y\right)(x)=\lambda\left(E_{\left(\rho_{1}^{(r)}\right), \ldots,\left(\rho_{m}^{(r)}\right) ; \beta_{1}, \ldots, \beta_{m} ; \omega_{r} ; 0_{+}}^{\left(\gamma_{r}\right),\left(l_{l}\right)}\right)(x)+f(x),
\end{aligned}
$$

If $\alpha, \beta_{j}, \gamma_{i}, l_{i}, \omega_{i} \in \mathbb{C} ; \mathfrak{R}(\alpha)>0 ; \mathfrak{R}\left(\rho_{j}^{(i)}\right)>0 ; \mathfrak{R}\left(\beta_{j}\right)>0$; $\Re\left(l_{i}\right)>0 ; j=1, \ldots, m ;$ and $i=1, \ldots, r$ with the initial condition $\left(I_{0+}^{1-\alpha} y\right)(0+)=c$ ( $c$ is an arbitrary constant $)$ and solution of differential equations existing in the space $L(0, \infty)$, then Theorems $2-4$ are stated in the following form.

then its solution is given by

Theorem 2. If

$$
\begin{aligned}
y(x)= & \frac{c x^{\alpha-1}}{\Gamma(\alpha)}+\lambda x^{\beta_{1}+\alpha} E_{\left(\rho_{1}^{(r)}\right), \ldots,\left(\rho_{m}^{(r)}\right) ; \beta_{1}+\alpha+1, \beta_{2}, \ldots, \beta_{m}}^{\left(\gamma_{r}\right),\left(l_{r}\right)}\left[\omega_{1} x^{\rho_{1}^{\prime}}, \ldots, \omega_{r} x^{\rho_{1}^{(r)}}\right] \\
& +\frac{1}{\Gamma(\alpha)} \int_{0}^{x}(x-t)^{\alpha-1} f(t) \mathrm{d} t .
\end{aligned}
$$


Theorem 3. If

then its solution is given by

$$
\begin{aligned}
\left(D_{0+}^{\alpha} y\right)(x)= & \lambda\left(E_{\left(\rho_{1}^{(r)}\right), \ldots,\left(\rho_{m}^{(r)}\right) ; \beta_{1}, \ldots, \beta_{m} ; \omega_{r} ; 0+}^{\left(\gamma_{r}\right),\left(l_{r}\right)}\right)(x) \\
& +p x^{\beta_{1}} E_{\left(\rho_{1}^{(r)}\right), \ldots,\left(\rho_{m}^{(r)}\right) ; \beta_{1}+1, \beta_{2}, \ldots, \beta_{m}}^{\left(\gamma_{r}\right),\left(l_{r}\right)}\left[\omega_{1} x^{\rho_{1}^{\prime}}, \ldots, \omega_{r} x^{\rho_{1}^{(r)}}\right],
\end{aligned}
$$

$$
y(x)=\frac{c x^{\alpha-1}}{\Gamma(\alpha)}+(\lambda+p) x^{\beta_{1}+\alpha} E_{\left(\rho_{1}^{(r)}\right), \ldots,\left(\rho_{m}^{(r)}\right) ; \beta_{1}+\alpha+1, \beta_{2}, \ldots, \beta_{m}}^{\left(\gamma_{r}\right),\left(l_{r}\right)}\left[\omega_{1} x^{\rho_{1}^{\prime}}, \ldots, \omega_{r} x^{\rho_{1}^{(r)}}\right] .
$$

Theorem 4. If

$$
x\left(D_{0+}^{\alpha} y\right)(x)=\lambda\left(E_{\left(\rho_{1}^{(r)}\right), \ldots,\left(\rho_{m}^{(r)}\right) ; \beta_{1}, \ldots, \beta_{m} ; \omega_{r} ; 0+}^{\left(\gamma_{r}\right),\left(l_{r}\right)}\right)(x),
$$

Proof. In Theorem 1, let the left-hand side of result (11) be $\Delta_{1}$, i.e.,

then its solution is given by

$$
\begin{aligned}
y(x)= & \frac{c x^{\alpha-1}}{\Gamma(\alpha)}+\frac{\lambda}{\Gamma(\alpha)} \int_{0}^{x} t^{\alpha-1}(x-t)^{\beta_{1}-1} \\
& \times E_{\left(\rho_{1}^{(r)}\right), \ldots,\left(\rho_{m}^{(r)}\right) ; \beta_{1}+1, \beta_{2}, \ldots, \beta_{m}}^{\left(\gamma_{r}\right),\left(l_{r}\right)}\left[\omega_{1}(x-t)^{\rho_{1}^{\prime}}, \ldots, \omega_{r}(x-t)^{\rho_{1}^{(r)}}\right] \mathrm{d} t .
\end{aligned}
$$

$$
\Delta_{1}=D_{a+}^{\alpha}\left[(t-a)^{\beta_{1}-1} E_{\left(\rho_{1}^{(r)}\right), \ldots,\left(\rho_{m}^{(r)}\right) ; \beta_{1}, \ldots, \beta_{m}}^{\left(\gamma_{r}\right),\left(l_{r}\right)}\left\{\omega_{1}(t-a)^{\rho_{1}^{\prime}}, \ldots, \omega_{r}(t-a)^{\rho_{1}^{(r)}}\right\}\right](x)
$$

Having used the definition of $E_{\left(\rho_{1}^{(r)}\right), \ldots,\left(\rho_{m}^{(r)}\right) ; \beta_{1}, \ldots, \beta_{m}}^{\left(\gamma_{r}\right),\left(l_{r}\right)}$ [.] given in (3), we obtain the following form:

$$
\Delta_{1}=\sum_{k_{1}, \ldots, k_{r}=0}^{\infty} \frac{\prod_{i=1}^{r}\left[\left(\gamma_{i}\right)_{l_{i} k_{i}}\left(\omega_{i}\right)^{k_{i}}\right]}{\prod_{j=1}^{m} \Gamma\left(\beta_{j}+\sum_{i=1}^{r} \rho_{j}^{(i)} k_{i}\right) \prod_{i=1}^{r}\left(k_{i} !\right)} D_{a+}^{\alpha}\left[(t-a)^{\beta_{1}+\sum_{i=1}^{r} \rho_{1}^{(i)} k_{i}-1}\right](x) .
$$

On using the fractional derivative of power function $(t-a)^{\beta_{1}+\sum_{i=1}^{r} \rho_{1}^{(i)} k_{i}-1}([20]$, p. 36, Equation $(2.26))$, we have

$$
\Delta_{1}=(x-a)^{\beta_{1}-\alpha-1} \sum_{k_{1}, \ldots, k_{r}=0}^{\infty} \frac{\prod_{i=1}^{r}\left[\left(\gamma_{i}\right)_{l_{i} k_{i}}\left(\omega_{i}\right)^{k_{i}}\right](x-a)^{\sum_{i=1}^{r} \rho_{1}^{(i)} k_{i}}}{\prod_{j=2}^{m} \Gamma\left(\beta_{j}+\sum_{i=1}^{r} \rho_{1}^{(i)} k_{i}\right) \Gamma\left(\beta_{1}-\alpha+\sum_{i=1}^{r} \rho_{1}^{(i)} k_{i}\right) \prod_{i=1}^{r}\left(k_{i} !\right)}
$$


On interpreting multiple series by the definition of $E_{\left(\rho_{1}^{(r)}\right), \ldots,\left(\rho_{m}^{(r)}\right) ; \beta_{1}, \ldots, \beta_{m}}^{\left(\gamma_{r}\right),\left(l_{r}\right)}[\cdot]$, we at once arrive at (11).

The proof of (12) follows the proof of (11) using (6) and ([20], p. 40, Equation (2.44)) therein.

Theorem 2 is proved as follows.

Use the definition of operator $\left(E_{\left(\rho_{1}^{(r)}\right), \ldots,\left(\rho_{m}^{(r)}\right) ; \beta_{1}, \ldots, \beta_{m} ; \omega_{r} ; a+}^{\left(\gamma_{r}\right),\left(l_{r}\right)} \psi\right)$ (x) (at $a=0$ and $\psi(x)=1$ ) and result (9) (at $\sigma=1$ ) in (13), we have

$$
\left(D_{0+}^{\alpha} y\right)(x)=\lambda x^{\beta_{1}} E_{\left(\rho_{1}^{(r)}\right), \ldots,\left(\rho_{m}^{(r)}\right) ; \beta_{1}+1, \beta_{2}, \ldots, \beta_{m}}^{\left(\gamma_{r}\right),\left(l_{r}\right)}\left[\omega_{1} x^{\rho_{1}^{\prime}}, \ldots, \omega_{r} x^{\rho_{1}^{(r)}}\right]+f(x) .
$$

Taking Laplace transform of (22) and then using formula (7) (for $n=1$ ) and (10), therein we have

$$
\begin{aligned}
& y(s)=c s^{-\alpha}+\lambda s^{-\beta_{1}-1-\alpha} \prod_{j=2}^{m}\left(\frac{1}{\Gamma\left(\beta_{j}\right)}\right)
\end{aligned}
$$

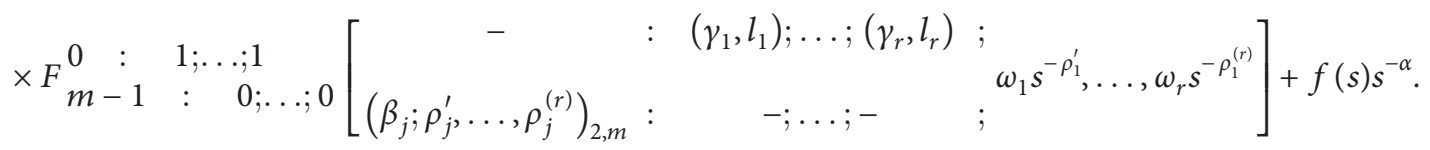

In view of the definition of generalized Lauricella function ([21], p. 37, Equations (21-23)), we have the form

$$
y(s)=c s^{-\alpha}+\lambda \frac{\sum_{k_{1}, \ldots, k_{r}=0}^{\infty} \prod_{i=1}^{r}\left[\left(\gamma_{i}\right)_{l_{i} k_{i}}\left(\omega_{i}\right)^{k_{i}}\right] s^{\left(-\beta_{1}-1-\alpha-\sum_{i=1}^{r} \rho_{j}^{(i)} k_{i}\right)}}{\prod_{j=2}^{m} \Gamma\left(\beta_{j}+\sum_{i=1}^{r} \rho_{j}^{(i)} k_{i}\right) \prod_{i=1}^{r}\left(k_{i} !\right)}+f(s) s^{-\alpha} .
$$

Applying inverse Laplace transform on both sides of (24) and using convolution theorem, we find

$$
\begin{aligned}
y(x)= & c \frac{x^{\alpha-1}}{\Gamma(\alpha)}+\lambda \sum_{k_{1}, \ldots, k_{r}=0}^{\infty} \frac{\prod_{i=1}^{r}\left[\left(\gamma_{i}\right)_{l_{i} k_{i}}\left(\omega_{i}\right)^{k_{i}}\right]}{\prod_{j=1}^{m} \Gamma\left(\beta_{j}+\sum_{i=1}^{r} \rho_{j}^{(i)} k_{i}\right) \prod_{i=1}^{r}\left(k_{i} !\right)} \frac{x^{\beta_{1}+\alpha+} \sum_{i=1}^{r} \rho_{j}^{(i)} k_{i}}{\Gamma\left(\beta_{1}+1+\alpha+\sum_{i=1}^{r} \rho_{j}^{(i)} k_{i}\right)} \\
& +\frac{1}{\Gamma(\alpha)} \int_{0}^{x} \frac{f(t)}{(x-t)^{1-\alpha}} \mathrm{d} t .
\end{aligned}
$$

Now, on interpreting the multiple series using (3), we at once arrive at desired result (14).
Proof. of Theorem 3. We use (at $a=0$ and $\psi(x)=1$ ) and (9) (at $\sigma=1$ ) in (15), and it takes the following form:

$$
\left(D_{0+}^{\alpha} y\right)(x)=(\lambda+p) x^{\beta_{1}} E_{\left(\rho_{1}^{(r)}\right), \ldots,\left(\rho_{m}^{(r)}\right) ; \beta_{1}+1, \beta_{2}, \ldots, \beta_{m}}^{\left(\gamma_{r}\right),\left(l_{r}\right)}\left[\omega_{1} \rho^{\rho_{1}^{\prime}}, \ldots, \omega_{r} x^{\rho_{1}^{(r)}}\right] .
$$


On both sides of (26), we take Laplace transform and then using formula (7) (for $n=1$ ) and (10) therein, we obtain

$$
\begin{aligned}
& y(s)=c s^{-\alpha}+(\lambda+p) s^{-\beta_{1}-1-\alpha}=\prod_{j=2}^{m}\left(\frac{1}{\Gamma\left(\beta_{j}\right)}\right)
\end{aligned}
$$

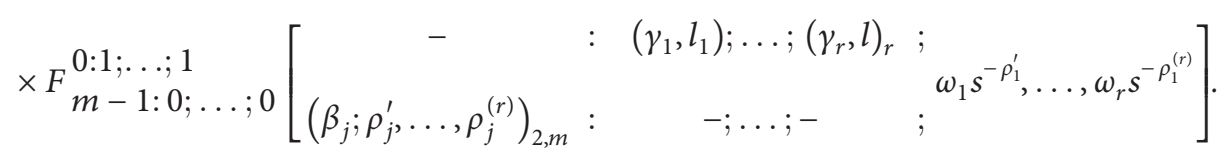

In view of the definition of generalized Lauricella function ([21], p. 37, Equations (21-23)), we have

$$
y(s)=c s^{-\alpha}+(\lambda+p) \sum_{k_{1}, \ldots, k_{r}=0}^{\infty} \frac{\prod_{i=1}^{r}\left[\left(\gamma_{i}\right)_{l_{i} k_{i}}\left(\omega_{i}\right)^{k_{i}}\right] s^{\left(-\beta_{1}-1-\alpha-\sum_{i=1}^{r} \rho_{j}^{(i)} k_{i}\right)}}{\prod_{j=1}^{m} \Gamma\left(\beta_{j}+\sum_{i=1}^{r} \rho_{j}^{(i)} k_{i}\right) \prod_{i=1}^{r}\left(k_{i} !\right)} .
$$

Applying inverse Laplace transform on (28), we have

$$
\begin{aligned}
y(x)= & c \frac{x^{\alpha-1}}{\Gamma(\alpha)}+(\lambda+p) \sum_{k_{1}, \ldots, k_{r}=0}^{\infty} \frac{\prod_{i=1}^{r}\left[\left(\gamma_{i}\right)_{l_{i} k_{i}}\left(\omega_{i}\right)^{k_{i}}\right]}{\prod_{j=2}^{m} \Gamma\left(\beta_{j}+\sum_{i=1}^{r} \rho_{j}^{(i)} k_{i}\right) \prod_{i=1}^{r}\left(k_{i} !\right)} \\
& \times \frac{x^{\left(\beta_{1}+\alpha+\sum_{i=1}^{r} \rho_{j}^{(i)} k_{i}\right)}}{\Gamma\left(\beta_{1}+1+\alpha+\sum_{i=1}^{r} \rho_{j}^{(i)} k_{i}\right)} .
\end{aligned}
$$

On interpreting the multiple series using (3), we at once arrive at result (16).

Proof. of Theorem 4. We use operator $\left(E_{\left(\rho_{1}^{(r)}\right), \ldots,\left(\rho_{m}^{(r)}\right) ; \beta_{1}, \ldots, \beta_{m} ; \omega_{r} ; a+}^{\left(\gamma_{r}\right),\left(l_{r}\right)} \psi(x)\right.$ (at $a=0$ and $\left.\psi(x)=1\right)$ and

(9) (at $\sigma=1)$ in (17), and we have the following form:

$$
x\left(D_{0+}^{\alpha} y\right)(x)=\lambda x^{\beta_{1}} E_{\left(\rho_{1}^{(r)}\right), \ldots,\left(\rho_{m}^{(r)}\right) ; \beta_{1}+1, \beta_{2}, \ldots, \beta_{m}}^{\left(\gamma_{r}\right),\left(l_{r}\right)}\left[\omega_{1} x^{\rho_{1}^{\prime}}, \ldots, \omega_{r} x^{\rho_{1}^{(r)}}\right] .
$$

On both sides of (30), we take Laplace transform and use formulae (8) and (10) (for $n=1$ ); then, we obtain

$$
\begin{aligned}
& \frac{d}{\mathrm{~d} s} y(s)+\frac{\alpha}{s} y(s)=-\lambda s^{-\beta_{1}-1-\alpha} \prod_{j=2}^{m}\left(\frac{1}{\Gamma\left(\beta_{j}\right)}\right) \\
& \times F_{m-1: 0 ; \ldots ; 0}^{0: 1 ; \ldots ; 1}\left[\begin{array}{ccc}
- & \left(\gamma_{1}, l_{1}\right) ; \ldots ;\left(\gamma_{r}, l\right)_{r} ; \\
\left(\beta_{j} ; \rho_{j}^{\prime}, \ldots, \rho_{j}^{(r)}\right)_{2, m}: & -; \ldots ;- & \omega_{1} s^{-\rho_{1}^{\prime}}, \ldots, \omega_{r} s^{-\rho_{1}^{(r)}}
\end{array}\right] .
\end{aligned}
$$


In view of the definition of generalized Lauricella function ([21], p. 37, Equations (21-23)), we have

$$
\frac{d}{\mathrm{~d} s} y(s)+\frac{\alpha}{s} y(s)=-\lambda \sum_{k_{1}, \ldots, k_{r}=0}^{\infty} \frac{\prod_{i=1}^{r}\left[\left(\gamma_{i}\right)_{l_{i} k_{i}}\left(\omega_{i}\right)^{k_{i}}\right]}{\prod_{j=2}^{m} \Gamma\left(\beta_{j}+\sum_{i=1}^{r} \rho_{j}^{(i)} k_{i}\right) \prod_{i=1}^{r}\left(k_{i} !\right)} s\left(-\beta_{1}-1-\alpha-\sum_{i=1}^{r} \rho_{j}^{(i)} k_{i}\right) .
$$

Since this is a linear differential equation of first order and first degree,

$$
y(s)=\lambda \sum_{k_{1}, \ldots, k_{r}=0}^{\infty} \frac{\prod_{i=1}^{r}\left[\left(\gamma_{i}\right)_{l_{i} k_{i}}\left(\omega_{i}\right)^{k_{i}}\right]}{\prod_{j=2}^{m} \Gamma\left(\beta_{j}+\sum_{i=1}^{r} \rho_{j}^{(i)} k_{i}\right) \prod_{i=1}^{r}\left(k_{i} !\right)} \frac{(s)\left(-\beta_{1}-\alpha-\sum_{i=1}^{r} k_{1} \rho_{1}^{(i)}\right)}{\left(\beta_{1}+\sum_{i=1}^{r} k_{i} \rho_{1}^{(i)}\right)}+c s^{-\alpha} .
$$

Taking inverse Laplace transform of (33), we have

$$
\begin{aligned}
y(x)= & \lambda \sum_{k_{1}, \ldots k_{r}=0}^{\infty} \frac{\prod_{i=1}^{r}\left[\left(\gamma_{i}\right)_{l_{i} k_{i}}\left(\omega_{i}\right)^{k_{i}}\right]}{\prod_{j=2}^{m} \Gamma\left(\beta_{j}+\sum_{i=1}^{r} \rho_{j}^{(i)} k_{i}\right) \prod_{i=1}^{r}\left(k_{i} !\right) 1 /\left(\beta_{1}+\sum_{i=1}^{r} k_{i} \rho_{1}^{(i)}\right)} \\
& \times \mathbf{Q}^{-1}[(s) \\
\left(-\alpha-\beta_{1}-\sum_{i=1}^{r} k_{1} \rho_{1}^{(i)}\right) & \left(c \boldsymbol{Q}^{-1}\left(s^{-\alpha}\right) .\right.
\end{aligned}
$$

In view of convolution theorem, we obtain

$$
\begin{aligned}
y(x)= & \frac{\lambda}{\Gamma(\alpha)} \sum_{k_{1}, \ldots k_{r}=0}^{\infty} \frac{\prod_{i=1}^{r}\left[\left(\gamma_{i}\right)_{l_{i} k_{i}}\left(\omega_{i}\right)^{k_{i}}\right]}{\prod_{j=2}^{m} \Gamma\left(\beta_{j}+\sum_{i=1}^{r} k_{i} \rho_{j}^{(i)}\right) \prod_{i=1}^{r}\left(k_{i} !\right)\left(1 / \Gamma\left(\beta_{1}+1+\sum_{i=1}^{r} k_{i} \rho_{j}^{(i)}\right)\right) .} \\
& \times \int_{0}^{x} t^{\alpha-1}(x-t)^{\beta_{1}-1+\sum_{i=1}^{r} k_{i} \rho_{1}^{(i)}} \mathrm{d} t+\frac{c}{\Gamma(\alpha)} x^{\alpha-1} .
\end{aligned}
$$

Now, on interpreting the multiple series in view of (3), we obtain the result in (18).

\section{Conclusion}

Here, we conclude further interesting known results:

(1) Our main results for $m=1$, respectively, give the known results provided by Gupta and Jaimini ([22], pp. 145-146, Equations (1-10)).

(2) If in result (10) and in Theorem 2, we take $r=1$ (i.e., $\omega_{2}=, \ldots,=\omega_{r}=0$ ), then result (10) reduces to the known result provided by Saxena et al.
([23], p. 10, Equation (50)) and Theorem 2 gives the correct form (at $v=0$ ) of the theorem provided by Saxena et al. ([23], p. 10, Theorem (5.1)).

(3) For $m=1$ and $r=1$, Theorems 1 to 4 reduce, respectively, to the known results (at $\nu=0$ ) provided by Srivastava and Tomovski [24].

(4) If in Theorem 1 , we take $m=1$ and $l_{1}=l_{2}=, \ldots,=l_{r}=1$, then these results, respectively, reduce to the known results provided by Gautam ([18], pp. 201-202, Equations (4.64)-(4.65)).

(5) If in Theorems 1 to 4 , we take $m=1$, $l_{1}=l_{2}=, \ldots,=l_{r}=1$, and $\rho_{1}^{\prime}=\rho_{1}^{\prime \prime}=, \ldots,=\rho_{1}^{(r)}=1$, 
then these, respectively, reduce to the results for function $\phi_{2}^{(r)}(\cdot)$ provided by Gupta ([25], pp. 250-253, Equations (4.9.19)-(4.9.27)).

Therefore, the results presented in the article would immediately yield a large number of results that include a wide range of special functions occurring in issues of scientific research, computer science, and applied mathematics, among others.

\section{Data Availability}

No data were used to support this study.

\section{Conflicts of Interest}

The authors declare no conflicts of interest regarding the publication of this article.

\section{References}

[1] H. M. Baskonus, "New acoustic wave behaviors to the DaveyStewartson equation with power-law nonlinearity arising in fluid dynamics," Nonlinear Dynamics, vol. 86, no. 1, pp. 177-183, 2016.

[2] H. M. Baskonus, "Complex surfaces to the fractional $(2+1)$ dimensional boussinesq dynamical model with local M-derivative," European Physical Journal Plus, vol. 134, no. 322, pp. 1-10, 2019.

[3] S. Dave, A. M. Khan, S. D. Purohit, and D. L. Suthar, "Application of green synthesized metal nanoparticles in the photocatalytic degradation of dyes and its mathematical modelling using the Caputo-Fabrizio fractional derivative without the singular kernel," Journal of Mathematics, vol. 2021, Article ID 9948422, 8 pages, 2021.

[4] H. F. Ismael, H. Bulut, H. Bulut, H. M. Baskonus, and W. Gao, "Dynamical behaviors to the coupled Schrödinger-Boussinesq system with the beta derivative," AIMS Mathematics, vol. 6, no. 7, pp. 7909-7928, 2021.

[5] W. Gao, P. Veeresha, D. G. Prakasha, B. Senel, and H. M. Baskonus, "Iterative method applied to the fractional nonlinear systems arising in thermoelasticity with MittagLeffler kernel," Fractals, vol. 28, no. 8, 2020.

[6] W. Gao, P. Veeresha, D. G. Prakasha, and H. M. Baskonus, "New numerical simulation for fractional Benney-Lin equation arising in falling film problems using two novel techniques," Numerical Methods for Partial Differential Equations, vol. 37, no. 1, pp. 210-243, 2021.

[7] H. Habenom, D. L. Suthar, D. Baleanu, and S. D. Purohit, “A numerical simulation on the effect of vaccination and treatments for the fractional hepatitis B model," ASME Journal of Computational and Nonlinear Dynamics, vol. 16, no. 1, Article ID 11004, 2021.

[8] A. Kumar, E. Ilhan, E. Ilhan, A. Yel, and H. M. Baskonus, "Extractions of some new travelling wave solutions to the conformable Date-Jimbo-Kashiwara-Miwa equation," AIMS Mathematics, vol. 6, no. 5, pp. 4238-4264, 2021.

[9] G. Yel, C. Cattani, H. M. Baskonus, and W. Gao, "On the complex simulations with dark-bright to the hirota-maccari system," Journal of Computational and Nonlinear Dynamics, vol. 16, no. 6, p. 9, Article ID 61005, 2021.

[10] K. S. Nisar, D. L. Suthar, R. Agarwal, and S. D. Purohit, "Fractional calculus operators with Appell function kernels applied to Srivastava polynomials and extended Mittag-Leffler function," Advances in Difference Equations, vol. 148, 2020.

[11] D. L. Suthar, H. Amsalu, and K. Godifey, "Certain integrals involving multivariate Mittag-Leffler function," Journal of Inequalities and Applications, vol. 2019, no. 1, p. 208, 2019.

[12] D. L. Suthar, H. Habenom, and H. Tadesse, "Generalized fractional calculus formulas for a product of Mittag-Leffler function and multivariable polynomials," International Journal of Applied and Computational Mathematics, vol. 4, no. 1, pp. 1-12, 2018.

[13] D. L. Suthar, M. Andualem, and B. Debalkie, "A study on generalized multivariable Mittag-Leffler function via generalized fractional calculus operators," Journal of Mathematics, vol. 2019, Article ID 9864737, 7 pages, 2019.

[14] R. K. Saxena and K. Nishimoto, "N-Fractional calculus of generalized Mittag-Leffler functions," Journal of Fractional Calculus, vol. 37, pp. 43-52, 2010.

[15] R. K. Saxena and K. Nishimoto, "Further results on the generalized Mittag-Leffler functions of fractional calculus," Journal of Fractional Calculus, vol. 40, pp. 29-41, 2011.

[16] V. S. Kiryakova, "Multiple (multiindex) Mittag-Leffler function related Gelfond and Leontiex operations and Laplace type integral transforms," Fractional Calculus and Applied Analysis, vol. 2, pp. 445-462, 1999.

[17] R. K. Saxena, S. L. Kalla, and V. S. Kiryakova, "Relation connecting multiindex Mittag-Leffler functions and Riemann-Liouville fractional calculus," Algebras Groups and Geometries, vol. 20, pp. 365-385, 2003.

[18] S. Gautam, Investigations in fractional differential operators of arbitrary order and their applications to special functions of one and several variables, Ph.D. Thesis, University of Kota, Kota, India, 2008.

[19] R. K. Saxena, S. L. Kalla, and R. Saxena, "Multivariate analogue of generalized Mittag-Leffler function," Integral Transforms and Special Functions, vol. 22, no. 7, pp. 533-548, 2011.

[20] S. G. Samko, A. A. Kilbas, and O. I. Marichev, Fractional Integrals and Derivatives: Theory and Applications, Gordon and Breach, New York, NY, USA, 1983.

[21] H. M. Srivastava and P. W. Karlsson, Multiple Gaussian Hypergeometric Series, Ellis Harwood Ltd. Chichestcra, New York, NY, USA, 1985.

[22] J. Gupta and B. B. Jaimini, "On certain fractional differential equations involving generalized multivariable Mittag-Leffler function," Note Mat, vol. 32, no. 2, pp. 141-156, 2012.

[23] R. K. Saxena, J. P. Chauhan, R. K. Jana, and A. K. Shukla, "Further results on the generalized Mittag-Leffler function operator," Journal of Inequalities and Applications, vol. 75, pp. 1-12, 2015.

[24] H. M. Srivastava and Ž. Tomovski, "Fractional calculus with an integral operator containing a generalized Mittag-Leffler function in the kernel," Applied Mathematics and Computation, vol. 211, no. 1, pp. 198-210, 2009.

[25] J. Gupta, Investigations in generalized special functions and the fractional calculus with its applications to univalent and multivalent functions, Ph.D. Thesis, University of Kota, Kota India, 2011. 